
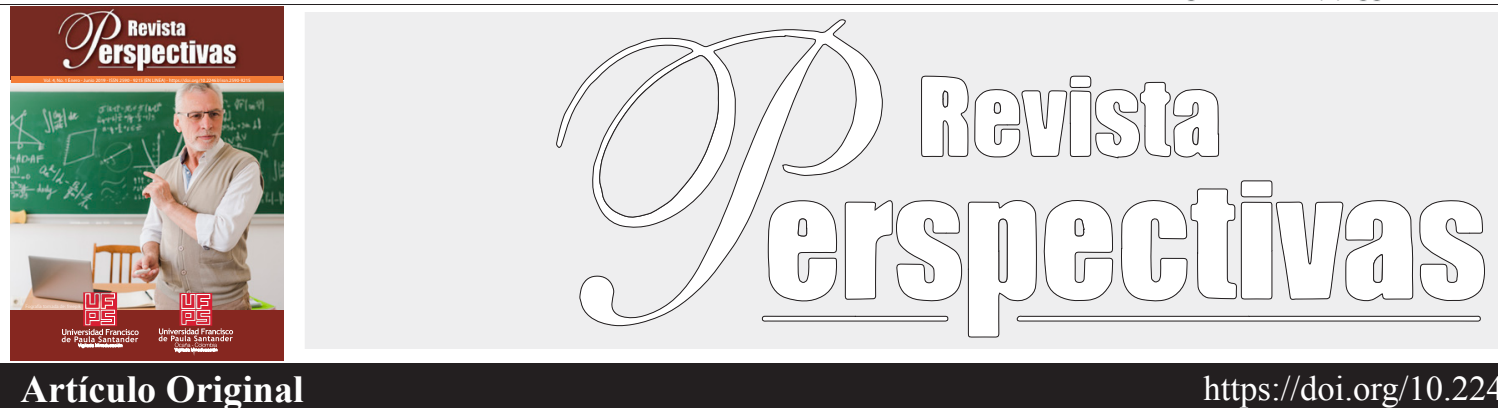

Artículo Original

https://doi.org/10.22463/25909215.1751

\title{
Agentes educadores y estereotipos sobre masculinidad: reflexiones para la formación de identidades masculinas alternativas
}

Educating agents and stereotypes about masculinity: reflections for the formation of alternative masculine identities Agentes educadores e estereótipos sobre masculinidade: reffexões para a formação de identidades masculinas alternativas.

Juan José Arias-Chamorro ${ }^{\mathrm{a}}$, Kathy Alejandra Navarro-Verjel ${ }^{\mathrm{b}^{*}}$, Laura Daniela Ortega-Garnica ${ }^{\mathrm{c}}$

a Magister en Educación, juanjoseach@ufps.edu.co, ORCID 0000-0002-6144-8082, Universidad Francisco de Paula Santander, Cúcuta, Colombia.

$b^{* *}$ Trabajadora Social (En formación), kathyalejandranv@ufps.edu.co, ORCID 0000-0002-5803-9632, Universidad Francisco de Paula Santander, Cúcuta, Colombia.

${ }^{c}$ Trabajadora Social (En formación), lauradanielaog@ufps.edu.co, ORCID 0000-0001-7324-4282, Universidad Francisco de Paula Santander, Cúcuta, Colombia.

Cómo citar: Arias, J. J., Navarro, K. A., \& Ortega, L. D. (2019). Agentes educadores y estereotipos sobre masculinidad: reflexiones para la formación de identidades masculinas alternativas. Perspectivas, 4(1), 14-22.

Recibido: Agosto 20, 2018; Aceptado: Noviembre 01, 2018

\begin{tabular}{ll}
\hline RESUMEN \\
\hline Palabras Clave: & Antecedentes. Estudios preliminares relacionados con el patriarcado, la hegemonía, las masculinidades alternativas y \\
& los contextos socializadores de representaciones acerca de lo masculino fortalecen el propósito de la revisión teórica \\
planteada. Objetivo. Analizar la construcción de la masculinidad en relación con la socialización de los diferentes agentes & educadores. Métodos. Se ha llevado a cabo una revisión sistemática de 50 documentos que abordan el tema "Masculinidad". \\
Escuela/colegio & Resultados. Se presentan la construcción de la masculinidad de acuerdo a la existencia de modelos tradicionales o nuevos, \\
Familia & así mismo de los espacios relacionales que socializan y reproducen el deber ser de hombres y mujeres, en esencia la \\
Masculinidad & familia, la escuela/colegio y la universidad. Conclusión. Se infiere que la masculinidad y su construcción se conceptualiza \\
Masculinidad hegemónica & desde la hegemonía y la alternatividad, siendo los agentes educadores transmisores culturales de lo que debe significar la \\
Nuevas masculinidades & masculinidad.
\end{tabular}

\section{ABSTRACT}

Keywords:

Background. Preliminary studies related to patriarchy, hegemony, alternative masculinities and socializing contexts of representations about the masculine strengthen the purpose of the theoretical review proposed. Objective. Analyze the

School/college

Family construction of masculinity in relation to the socialization of the different educating agents. Methods. A systematic review of 50 documents that address the topic "Masculinity" has been carried out. Results. The construction of masculinity is presented according to the existence of traditional or new models, as well as the relational spaces that socialize and

Masculinity

Hegemonic masculinity

New masculinities

University reproduce the duty to be of men and women, in essence the family, the school / college and the university. ${ }^{\circ}$ It is inferred that masculinity and its construction is conceptualized from the hegemony and the alternative, being the educating agents cultural transmitters of what masculinity should mean.

\begin{tabular}{ll}
\hline & RESUMO \\
\hline Palavras chave: & $\begin{array}{l}\text { Antecedentes. Estudos preliminares relacionados ao patriarcado, hegemonia, masculinidades alternativas e contextos } \\
\text { socializadores de representações sobre o masculino reforçam o objetivo da revisão teórica proposta. Objetivo. }\end{array}$ \\
Escola/faculdade & $\begin{array}{l}\text { Analisar a construção da masculinidade em relação à socialização dos diferentes agentes educadores. Métodos. Uma } \\
\text { revisão sistemática de } 50 \text { documentos que abordam o tema "masculinidade" foi realizada. Resultados. A construção } \\
\text { Família }\end{array}$ \\
da masculinidade é apresentada de acordo com a existência de modelos tradicionais ou novos, assim como os espaços \\
Masculinidade & relacionais que socializam e reproduzem o dever de ser homem e mulher, em essência a família, a escola / faculdade e \\
Novas masculinidades & a universidade. Conclusão. Infere-se que a masculinidade e sua construção são conceituadas a partir da hegemonia e da \\
Universidade & alternativa, sendo os agentes educadores os transmissores culturais do que a masculinidade deve significar.
\end{tabular}

*Autor de Correspondencia

E-mail: kathyalejandranv@ufps.edu.co (Khaty Alejandra Navarro)

(c) $($ (i) $\ominus$ Peer review is the responsibility of the Universidad Francisco de Paula Santander 


\section{Introducción}

Este artículo parte de una revisión teórica que pretende analizar la forma como se construye socialmente la masculinidad a través de las diferentes instituciones educadoras y como éstas inciden frente a lo que significa ser, pensar, sentir y hacer sobre lo masculino. Así mismo explora como los distintos agentes formadores: familia, escuela-colegio y universidad, contextos sociales, culturales y académicos edifican las prácticas y los imaginarios sobre el género. En cuanto al núcleo familiar, es visto como el primer ambiente que socializa patrones culturales, comportamientos y actitudes frente a la manera de "ser hombre" siendo ejemplo papá y mamá, así como todas las figuras femeninas y masculinas de la familia.

Por otro lado, la escuela es otra institución que desde los espacios educativos o lúdicos permite visualizar las diferentes formas de la masculinidad, permitiendo reforzar o transformar la identidad masculina a partir de características o atributos que pueden denotar tradición o por el contrario nuevas maneras de actuación del hombre, aquí son ejemplos para dicha construcción social los grupos de amigos y docentes. Finalmente, también se considera como un agente socializador la universidad, siendo esta un espacio académico que tiene como reto la formación profesional y personal de los estudiantes, representa un entorno en donde se construye y deconstruye lo masculino; aquí hombre y mujer deben repensar su manera de actuar y ser consigo mismo, con el otro, con las mujeres y con los mismos hombres.

\section{Materiales y métodos}

La búsqueda bibliográfica se realiza mediante fuentes de información primarias, secundarias y terciarias como revistas con publicaciones originales de estudios científicos, artículos de revisión o reflexión, y compendios, de manera que para la recolección de estos se han seleccionado las bases de datos: EBSCO host, SciELO, Redalyc, Dialnet y la Revista Perspectivas. Así mismo, la estrategia para la búsqueda se documenta a través de seis términos entre ellos: masculinidad, masculinidad hegemónica, nuevas masculinidades, familia, escuela/colegio y universidad, hallándose así una cantidad de 50 referentes. Los artículos que se han seleccionado se orientan por el objetivo de la revisión, por otra parte, también se considera el desarrollo teórico y metodológico de los mismos e igualmente el grado de cientificidad teniendo en cuenta que son documentos extraídos de bases de datos confiables.

\section{Resultados y Discusión}

La Masculinidad: ¿Asignación biológica o construcción social?
La masculinidad es entendida como un conjunto de prácticas, comportamientos, valores y funciones que la sociedad atribuye de manera natural o esencial al varón, caracterizada por cualidades como la virilidad y la fuerza. Por lo tanto, cuando se habla de masculinidad se debe tener en cuenta aspectos como la vida familiar, los factores sociológicos y el lugar de origen, estas facetas inciden en la construcción de la identidad masculina del mismo modo que la vida sexual de los varones, al ser considerada un aspecto fundamental en las historias de vida de los hombres, el color de la piel influye de manera significativa en las vivencias sexuales del varón, esta particularidad ofrece ventajas e inconvenientes a hombres negros y hombres blancos según el contexto social (Castaño, 2011). Puede decirse que un elemento distintivo de la masculinidad es la fuerza física, sin embargo, lo sexual también entra a ser considerado un tema eminentemente masculino y así mismo, muchos jóvenes conceden gran significado a las relaciones con sus pares (amigos y amigas) puesto que el tiempo que pasan con estos, influye en la construcción de su identidad. Asocian que lo femenino pertenece a las mujeres y lo masculino a los hombres y que ellas deben mantenerse al margen de conductas masculinas y ellos de características femeninas (Matamala y Rodríguez, 2010).

En ese sentido, la masculinidad es un constructo histórico y cultural, lejos de aquel determinismo biológico que generaliza una particular y única forma de ser hombre, las concepciones y las prácticas sociales en torno a este concepto varían según los tiempos y lugares (Téllez y Verdú, 2001). De allí que sea importante entender cómo se concibe el "ser hombre", Martino (2013) afirma al respecto, que es poseer un cuerpo caracterizado por genitales masculinos, esta interpretación inverosímil vincula atributos físicos a una identidad personal sin prestar atención a las interacciones sociales. En relación con la historia, la noción de hombre se ha caracterizado por el grado de poder que posee, creando una manera popular de ser varón y repetitiva coadyuvada por la religión, los medios de comunicación, la estructura salarial y otros tantos. profundizando una masculinidad hegemónica como un modelo de práctica que legitima, produce y reproduce el dominio sobre mujeres y sobre otros hombres en un sistema patriarcal. Además ha promovido el desarrollado de otro tipos de masculinidades, la conservadora y la subordinada, la primera, aunque no responde a ese ideal de hombre antes señalado, si permite que ese sistema de dominio se mantenga, debido a que les ofrece beneficios por el hecho de pertenecer al colectivo hombres y, la segunda, que se caracteriza por desarrollarse entre grupos de hombres en donde se manifiestan relaciones de poder a través de la dominación y subordinación especialmente de varones heterosexuales a hombres homosexuales.

Este panorama lleva a discutir sobre la construcción de la masculinidad que de acuerdo a Otegui (1999) se puede resumir en tres problemáticas; las maneras en que las personas integran y adoptan las características que socialmente se edifican 
sobre el sexo; las diferentes construcciones de sexo- género relacionadas con los entornos socio- históricos que permiten su propagación; y la necesidad de exponer la existencia de identidades diversas que se edifican desde una perspectiva relacional. Así mismo, esta discusión tiene presente que la masculinidad refleja transformaciones culturales, acciones o prácticas relacionadas con el pasado, jerarquías en las relaciones entre hombres y mujeres, definiciones según grupos sociales, y formas de representación basadas en estereotipos o maneras alternativas (Hernández, 2010).

Aquí, conviene distinguir que, la masculinidad es una construcción social al igual que la feminidad que se da en cierta parte por los comportamientos que establecen los diferentes entornos sociales, al ser los colegios y universidades algunos de estos contextos se plantea la necesidad que desde los planes de estudio de las diferentes enseñanzas y con ayuda del o la docente se apoye la diferenciación de ciertos términos, entre ellos sexo, género, identidad de género, sexualidad y expresión de género (Sagaró y Moraga, 2015). Es cierto que algunas construcciones de la masculinidad proyectan características como la violencia, de acuerdo a Federici (Citado en Oliveras, Vigo, Johnson, Rivera, y Silva, 2018) hay varias maneras de ejercer violencia en el sistema capitalista patriarcal, pues es un modelo social y económico que se caracteriza por ser sexista y racista al realizar juicios despectivos a quienes se consideran débiles. Si se habla de la violencia de género, por ejemplo, López (2013) refiere que se instala y mantienen en el modelo de masculinidad tradicional, porque los roles de hombres y mujeres son rígidos y absolutos, aquí la igualdad es vista como un factor de riesgo para las definiciones de las identidades femeninas y masculinas establecidas.

En general, en relación con el constructo social de la masculinidad siguen existiendo en la familia y en los procesos educativos patrones hegemónicos, donde el hombre se caracteriza por ser dominador, lo que genera conflictos entre parejas y familias (Vendrell, 2002). De manera puntual se deduce que esa visión tradicional de lo masculino debe transformarse edificando relaciones igualitarias y equitativas, donde se construyan nuevas configuraciones de la masculinidad que reflejan cambios en las relaciones entre géneros, es de añadir que aunque la construcción de esta categoría se relaciona con los hombres es imprescindible también involucrar a las mujeres en ella teniendo en cuenta que lo masculino es construido por las conciliaciones entre hombres y mujeres (Gutmann, 2002). La política pública para la fragilidad humana expuesta en el ensayo de Díaz (2014) es un claro ejemplo del papel tan importante que tienen las mujeres en la construcción del género masculino, debido a que desde el hogar como ambiente privado derriban estereotipos que impiden al hombre ejercer las capacidades de ternura, cuidado y caricia, siendo su propósito ceder espacio a las nuevas masculinidades.

Cabe señalar que las identidades femeninas y masculinas se están redefiniendo desde discursos, prácticas o acciones que culturalmente se han aceptado, al hablar de la identidad masculina y su formación se afirma que cada hombre es quien se construye como sujeto masculino a partir del desarrollo o no de acciones, cada uno reconstruye patrones de acuerdo a lo que sea mejor para sí mismo (Macías, 2014). Por lo tanto, no debe hablarse de masculinidad sino de masculinidades, las cuales pueden ser diversas, diferentes o contrarias. Siguiendo este planteamiento Brod, Mac An Ghaill, Rotundo, Kimmel, y Bly (citado en Minello, 2002) subrayan que existen diferentes masculinidades, algunas se originan en relación con otras o por el contrario a veces conviven, siendo unas más aceptadas que otras, una de ellas es la hegemónica caracterizada por prácticas que simbolizan una postura de superioridad del hombre y de inferioridad en las mujeres, no obstante, este modelo no cohíbe la existencia de prácticas nuevas o subalternas.

Dos de las funciones del modelo hegemónico señaladas por Demetriou (citado en Díez, 2015) son; una hegemonía externa, es decir una dominación masculina sobre la mujer, y una hegemonía interna, que se da por una distinción de unos hombres sobre otros, de manera que no se desarrolla subordinación solo sobre lo femenino sino también sobre otro tipo de masculinidades. Es importante mencionar, que ésta comprende el cúmulo de comportamientos que asimilan la mayoría de los varones en países occidentales, además, de concebirse completamente opuesta a lo femenino, en ese sentido es el punto de comparación para valorar otras maneras de vivir la masculinidad.

Por consiguiente, ser masculino implica ser en primera medida heterosexual, en segundo lugar, ponderación del cuerpo del hombre y neutralidad o silencio afectivo (Ceballos, 2012). En ese sentido, algunos de los imaginarios de hombres y mujeres que sustentan el modelo hegemónico son en general el rechazo a la expresión de sentimientos y emociones; el uso de la fuerza hacia otros como algo que es natural en los hombres; una sexualidad que debe ser potente y que permita preñar a la mujer, establecer un hogar y tener autoridad sobre la familia, en particular este modelo se mantiene y apoya en la familia, la escuela y los medios de comunicación, es por ello necesario el establecimiento de espacios en la academia que permita sensibilizar, formar y promover nuevas maneras de ser hombre (Sandoval, 2014).

\section{¿Nuevos tiempos significa nuevos hombres?}

Aunque el modelo hegemónico aún esté presente en sociedades y en los contextos diarios, actualmente ha surgido una nueva masculinidad, que cuestiona y crítica todas esas formas de ser hombre basadas en el machismo y en las relaciones desiguales, las formas masculinas emergentes son practicadas por hombres que difieren de los tipos de violencia que se ejercen sobre otros, replantean la atribución que debe tener el hombre por ejemplo sobre la paternidad y sobre prácticas de salud sexual y reproductiva, cabe agregar que 
estos grupos suelen ser conformados por universitarios, lo que representa una limitación y un sesgo en la transformación masculina, primero porque no se logra involucrar a la población popular y además porque los integrantes de estos colectivos son discriminados y señalados por considerarse asociaciones homosexuales (Bard, 2016). De acuerdo a lo anterior, Boscán (2006) afirma, que replantear la masculinidad no significa solo una transformación actitudinal y mental sino un cambio en todos los ámbitos de la vida, aspectos económicos, políticos, sociales y culturales, para llegar a esa masculinidad positiva se deben cuestionar los modelos hegemónicos y promover el desarrollo de masculinidades múltiples reconociendo que es primordial la participación de hombres y mujeres en la instauración de las nuevas expresiones.

Avanzando en este ejercicio, aparece un nuevo concepto "nuevas masculinidades positivas" que unifica varias maneras de percibirse hombre y evidencia comportamientos diferentes, nuevos y que se catalogan como positivos al ir en contra de la homofobia y del machismo, en este modelo se presentan condiciones de carácter sociopolítico e ideológico, algunas de las primeras son el dejar la figura de dominador, apropiarse de las labores del hogar y la crianza de los hijos, reunirse con otros hombres y repensar su ser; por su parte las segundas sugieren dejar de promocionar todo tipo de violencia, comprender que existen diversos tipos de masculinidad y definirse a sí mismos teniendo en cuenta que la heterosexualidad no es la única forma de concretar su vida social (Boscán, 2008). Así, por ejemplo, surgen agrupaciones de hombres que buscan la equidad entre género y que rechazan todo tipo de poder ejercido hacia mujeres y hacia los mismos hombres con el fin de construir una sociedad más justa, conocidos en América Latina como "Hombres por la igualdad" (Soto, 2015).

Por ende, se puede afirmar que, si existen nuevas masculinidades y que estas han empezado a emerger, transformando a su paso las tradiciones hegemónicas, ya no existe una única forma de ser varón y a pesar de que muchos grupos sociales aún tienen como punto de referencia las masculinidades antañas, se ha ido evidenciando cambios en los paradigmas de género (Sanfélix, 2011). Para ello se requieren estrategias de formación dirigidas a hombres que permitan reflexionar sobre los patrones culturales del patriarcado y que de paso sean una alternativa para la difusión de los derechos humanos y la prevención de la violencia hacia las mujeres y hacia los mismos hombres (Ariza, Gaviria, Geldres y Vargas, 2015).

Estas nuevas prácticas pueden llegar a tener un impacto social importante, ya que se estaría reemplazando un sistema hegemónico cuyas consecuencias son el acoso y la violencia de género, conviene subrayar que, en la construcción de nuevas masculinidades, se deben crear espacios de diálogo que consientan la reflexión de nuevos referentes masculinos (Ríos, 2015). Estos avances en materia de género están inmersos en un proceso de cambio cultural afectados por concepciones del pasado y del presente, que luchan por superar un sistema patriarcal, efectivamente están aconteciendo nuevas prácticas sociales que golpean la esencia de las sociedades tradicionales (Montesinos, 2002).

Otro rasgo importante surge desde la perspectiva de género, que en conjunto con la ética se encarga de deconstruir normas morales y establecer quienes fueron los autores de dichos fundamentos, mencionando además quienes estuvieron excluidos de su participación, a través del análisis se obtuvo que las mujeres y muchos hombres habían sido apartados de la conceptualización de códigos morales que influían en su cotidianidad, así mismo el enfoque de género permite mostrar la construcción relacional de la identidad masculina y la identidad femenina, repensar aspectos como el ejercicio de poder en todos los contextos, mejorar la calidad de vida de mujeres y hombres en cuanto a derechos humanos y cuestionar las normatividades excluyentes, jerárquicas y sexistas, que han mantenido estas divisiones entre los géneros, si se cimienta desde abajo dichos elementos seguramente se va a lograr relaciones humanas equitativas e iguales (Figueroa, 2016).

\section{La familia en la construcción de la masculinidad}

La familia es el espacio primario donde se efectúa la socialización de normas y valores que rigen a los individuos, desde muy temprano el núcleo familiar va incitando un sistema de diferenciación entre ambos sexos, las reglas sociales van trazando las expectativas con respecto a los roles que hombres y mujeres deben asumir, de modo, que la idea que se tiene de padre, madre, esposo o esposa está condicionada por la sociedad de la cual somos el resultado (Herrera, 2000).

En el ámbito familiar el padre y la madre son figuras que tienen roles diferentes, aunque se espera que el padre asuma la presencia y participación en el desarrollo integral de los hijos, sin embargo, la historia ha demostrado que es la mujer quien confronta la crianza continua (Otálora y Mora, 2014). De manera que, a partir del espacio doméstico y también productivo los roles o funciones de hombres y mujeres se pueden fijar desde relaciones de género tradicional, de ayuda o de respeto mutuo (Muñoz citado en Gallego, 2018). Es por ello que, la familia es un agente educativo y también de cambio en la medida en que todos sus miembros se involucren al reconstruir las formas en que se regulan u organizan, de tal modo que establezcan arquetipos masculinos y femeninos basados en la democracia y la igualdad, donde las responsabilidades y funciones al interior de ella se distribuyan equitativamente (Hervías, 2015).

De acuerdo a lo anterior, específicamente con la organización familiar y añadiendo el desempleo masculino, se puede afirmar que los roles al interior del hogar no cambian significativamente, pues aunque la mujer asuma el rol de proveedor económico, el hombre no asume las tareas domésticas como función principal, solo las admite como complementarias, lo hace especialmente para compensar el esfuerzo de su pareja, 
no las acepta en su totalidad porque manifiesta que no fue educado para ello, de manera que piensa que ese rol no le corresponde (Martín y Echavarría, 2017). Dicho así, el ámbito familiar continúa siendo el principal, aunque no el único escenario donde se manifiesta, se reproduce y se transmite el deber ser de hombres y mujeres, lamentablemente aún permanecen los valores sexistas y machistas que minusvaloran las capacidades y labores femeninas en relación a lo masculino (Barba y Gómez, 2016).

La familia se establece además como un espacio fundamental en el que se construye el significado de ser hombre y ser mujer e influye en cómo deben vivir la sexualidad, siendo la primera y principal instancia de socialización en la que se transmiten y producen patrones culturales, allí jóvenes hombres y mujeres se atribuyen roles, un hombre puede o no realizar ciertas actividades, la influencia del padre y la madre es tal que aunque los jóvenes intentan vivir sus experiencias de manera propia resulta siendo difícil, ya que la autoridad de los padres en particular hace que se les obedezca, la mujer es mucho más limitada al momento de ejercer su sexualidad en este contexto y aunque el varón es consciente de las desigualdades construye y acepta estos esquemas (Martínez y Solís, 2009).

Por lo que se refiere a la formación de la identidad, es importante compartir que las relaciones con el padre y la madre tiene su influencia pues de manera general ellos se comportan conforme a patrones culturales existentes en un periodo o lapso de tiempo, por lo tanto, los niños y niñas perciben las maneras en que deben comportarse respecto a su sexo (Carrillo y Revilla, 2006). Siguiendo a Ospina (2007) y su trabajo sobre las Representaciones sociales de masculinidad y su expresión en el ámbito familiar, se evidencia que son características del hombre la autoridad, la fuerza física para trabajos considerados pesados y la fuerza mental reconocida como la capacidad para tomar las mejores decisiones dentro de la familia, también son atributos del varón proveer económicamente a su mujer e hijos, a su vez debe estar alejado de las emociones, de lo expresivo, incluso no puede ser empático y no debe tener miedo. Como se puede deducir todas estas cualidades van de la mano con el modelo tradicional de la masculinidad, siendo relevante incluir atributos que son considerados necesarios para alcanzar relaciones de género basadas en la igualdad y la justicia.

\section{La escuela o colegio en la construcción de la masculinidad}

La masculinidad se construye a través de procesos de aprendizaje culturales y sociales mediante relaciones de género y sexo, por lo tanto, se entiende que al ser un proceso construido son ineludibles los contextos donde un hombre y una mujer nacen, aprenden y se relacionan, uno muy importante es el sistema educativo, un agente socializador que instaura ideas y concepciones alrededor de los roles de género, en él está inmerso la actividad física y el deporte, estos dos factores han normalizado una noción de ser hombre basada en la fuerza, la habilidad y la capacidad física que debe poseer el varón, lo que de manera subliminal fortalece un sistema patriarcal, este prototipo de masculinidad subordina, excluye y discrimina lo que no se amolda a sus patrones (Vidiella, Herraiz, Hernández y Sancho, 2010).

La función que tiene la escuela en la construcción de la identidad masculina es la divulgación de conocimientos, virtudes y por supuesto maneras de actuar, se propagan ideologías liberadoras o no en la edificación de identidades (Sancho, Hernández, Herraiz y Vidiella, 2009). Algunas características masculinas reproducidas por la escuela son "la fortaleza física, a la viveza, a la irresponsabilidad y al abuso físico contra los más débiles y contra las mujeres" (Callirgos citado en Sancho et al., 2009, p.1168). De esta manera al ser las instituciones de índole educativo agentes socializadoras de las cuestiones relacionadas con el género, se definen como espacios estratégicos para la intervención sobre las masculinidades (Bell Hooks citada por Sancho et al., 2009).

De esta manera, la escuela constituye un espacio simbólico de intercambios ocupado por estudiantes en el que sus comportamientos con respecto a sus compañeros son un reflejo de las conductas y cualidades pertenecientes a patrones dominantes, para ellos su válida como varones reside en que tanto poder pueden ejercer contra las mujeres y contra otros (Lomas, 2013). Como se ha dicho los centros educativos además de transmitir conocimientos, reproduce estereotipos de género y fundamenta la construcción de las identidades, es decir difunde normas, principios, reglas y valores (Sancho, Hernández, Herraiz, y Vidiella, 2009).

En la educación pre-escolar por ejemplo, se producen acciones que representan aspectos de la masculinidad, una de estas son las agresiones físicas de los varones a otros niños orientadas para ejercer dominio; tales agresiones para las niñas son vistas como protectoras y amenazantes, además estas actuaciones son reprobadas por el docente o en algunos casos legitimadas, en consecuencia, la indisciplina toma significado en la construcción de la identidad masculina, es por ello que el sistema educativo es crucial para la trasformación de este tipo de símbolos siendo requerida la formación con perspectiva de género en los docentes de educación básica (Tomasini, 2010).

Otra clara muestra de la influencia de la escuela en la formación de la identidad masculina son los patios, los cuales se consideran espacios varoniles donde se visualiza que las interacciones entre ambos géneros es poca; como resultado aparece el debate de los docentes por intervenir o no sobre las dinámicas sexistas en los recreos y la manifestación de las familias de que se invierta en la coeducación para la formación en equidad (Garay, Vizcarra y Ugalde, 2017). Sobre la coeducación Gordillo (2007) expresa que es la búsqueda de la igualdad entre hombres y mujeres mediante su interacción, sin embrago, ve en la formación diferenciada en los sexos mejores rendimientos académicos, además defiende que estas 
diferencias se relacionan con aspectos profundos y no del exterior que hacen que hombres y mujeres busquen su propia manera de ser.

Hay que mencionar además, que la cultura está inmersa en la construcción de género, un espacio que no se ha analizado aún es el rural, la investigación desarrollada por Contreras \& Ramírez (2012) en torno a la masculinidad hegemónica en niños de una escuela rural en chile, sintetiza como la representación de esta no difiere de los significados que se expresan en otros contextos, es decir, en este ámbito también se observan patrones como la jerarquización, los hombres por sobre las mujeres y el hombre fuerte por sobre el hombre débil.

En definitiva, las instituciones educativas son medios fundamentales para transmitir la cultura de generación en generación y su influencia tiene una conmoción importante en el desarrollo del ser humano, es por esto que los educadores deben reflexionar sobre su labor y el trabajo que tiene la escuela como institución y agente socializador en temas de género (Connell, 2001). A modo de ejemplo el proyecto Educación para el presente sin violencia: construir una cultura de paz, constituye una herramienta pedagógica útil y de transformación social ante la divergencia de géneros, pues al ser un programa para la prevención de género implementado en instituciones educativas demuestra que, si es posible cambiar las formas de expresión de violencia de género, reduciéndolas en la cotidianidad (Barragán y González, 2007).

\section{La universidad en la construcción de la masculinidad}

La universidad según Cerva (2018) es un espacio que permite derrumbar y reconstruir la identidad de los hombres, esta institución advierte que los valores y habilidades que no se orientan a la equidad de género se vinculan con el establecimiento de rangos "ligados con el prestigio, la idea de mérito, competencia, autoridad científica, etc.” (p.44). Como lo refiere en su estudio Cubillas, Abril, Domínguez, Román, Hernández y Zapata (2016) el nivel de escolaridad puede incidir en la transición a idearios menos estereotipados de género, a su vez los jóvenes universitarios viven en medio de la modernidad y el arraigo de las costumbres, la población estudiada de jóvenes universitarios avanza poco a poco en concepciones sobre género hacia unas prácticas e ideas de mayor equidad, al menos en el discurso, es importante señalar que en los varones la deconstrucción es pausada debido a las ventajas que un sistema como el hegemónico les ofrece. Es por esto que los hombres no desean abandonar los patrones tradicionales de la masculinidad, este sistema les proporciona poder considerándolos más importantes (Mardones y Vizcarra, 2017).

Todavía cabe señalar que pese a las semejanzas que existen entre jóvenes universitarios en relación a su nivel de escolaridad, a la pertenencia a una misma generación y clase social, existen importantes diferencias en las concepciones y prácticas que tienen respecto a la masculinidad, esto debido a las articulaciones institucionales que se dieron a lo largo de su vida entre la escuela y la familia. Entre aquellos jóvenes que vivieron en familias más tradicionales, que fueron educados en valores religiosos y se insertaron en ambientes escolares más jerárquicos, encontramos las posturas y formas de relación más conservadoras. Quienes vivieron en familias con estructuras y formas de organización más equitativas, se educaron en valores laicos y se insertaron en ambientes escolares orientados democráticamente, establecen relaciones menos asimétricas con las mujeres y tienden a ser más flexibles respecto a los roles masculinos y femenino. Así mismo, se hizo notorio que la inserción en el ambiente universitario tiene una influencia importante para reforzar o modificar sus concepciones respecto a los modelos dominantes de masculinidad. Esto obliga a pensar en el importante papel que tienen las instituciones de educación superior para socializar modelos de masculinidad que promuevan relaciones más equilibradas y formas de convivencia más justas entre hombres y mujeres (Guevara, 2006).

En relación a un estudio realizado por Vázquez y Castro (2009) con estudiantes de la Universidad Autónoma Chapingo se encuentra que la masculinidad desde esta institución educativa plantea el requisito de hacer uso de la violencia y consumo de alcohol para mostrarse a los demás como varones, por otra parte, también establece la necesidad de abandonar estas dos conductas finalizando la vida universitaria para tomar responsabilidades. En síntesis, la masculinidad en la universidad se revela a través de la violencia de estudiantes mayores hacia los más jóvenes y hacia los homosexuales, también desde el consumo del alcohol para mejorar relaciones con los amigos evidenciando su masculinidad y alejándose de lo expresivo, no obstante existe una construcción que critica los patrones tradicionales, por ejemplo, existen hombres que debaten sobre la seducción como tarea solo de los hombres o que para ser hombres es necesario acostarse con muchas mujeres (Vázquez y Castro, 2009).

Otra interesante investigación es la que se desarrolla con dos grupos de hombres de Buenaventura en relación a los significados sobre la masculinidad, dejando entrever que los hombres del barrio Seis de Enero reproducen unas connotaciones tradicionales que representan la masculinidad hegemónica, reafirmando de esa forma la cultura patriarcal dominante de las comunidades afro de la costa pacífica colombiana, territorio en el que se valora la virilidad y la sexualidad como cualidades esenciales del hombre, asimismo, se reconoció un modelo de masculinidad emergente o nueva masculinidad por parte de ciertos estudiantes de arte dramático de la Universidad del Valle, para estos hombres la biología no determina la sexualidad y aceptan los roles de género como construcciones sociales, es evidente que las diferencias en las construcciones simbólicas alrededor de la masculinidad por 
parte de ambos grupos de hombres, están relacionadas con un mayor nivel de escolaridad (Rodríguez y Solano, 2018).

\section{Conclusiones}

La masculinidad se construye alrededor de parámetros culturales que han sido aceptados por hombres y mujeres, los cuales se transforman en determinado tiempo y espacio, existen modelos tradicionales que aceptan características como la dominación, la rudeza y la superioridad ante las mujeres o por el contrario modelos alternativos que reflejen nuevas maneras de comportamiento equitativas y empáticas de los varones con las mujeres y otros hombres.

Es cierto que agentes como la familia, la escuela y la universidad socializan las maneras como hombres y mujeres deben comportarse, cabe señalar que estas instituciones educadoras comparten lo que socialmente es aceptado, de manera que en lo que respecta a la construcción de las identidades es inevitable emplear estereotipos de género. Una de las propuestas para evitarlos implica que cada uno de estos agentes brinde la oportunidad de visualizar nuevos modos de ser, teniendo en cuenta que cada época trae consigo diferentes cambios a nivel cultural.

Hombres y mujeres son como son, como consecuencia de aspectos culturales tales como el seno familiar, la instrucción escolar, el grupo de iguales, los medios de comunicación, el lenguaje, el ámbito económico, la sociedad inmediata y con ella tradiciones, estilos de vida y creencias que influyen decisivamente en la construcción de las identidades humanas. Dicho de otro modo, las personas nacen con un sexo y a este se le añade las formas de ser hombre y mujer de una sociedad determinada, por esta razón las identidades masculinas y femeninas no son solo un acontecimiento biológico y natural sino también, un proceso cultural, es así como, hombres y mujeres son diferentes no sólo porque tengan un sexo inicial distinto sino también porque aprenden a ser de unas determinadas maneras

Sibien existen en cada contexto educador formas diferentes de actuar en cada hombre y mujer también se evidencia que se reproducen comportamientos y atributos hegemónicos con respecto a la construcción de la masculinidad, esto significa que el modelo dominante no se ha derrumbado por el contrario impera al interior de cada sujeto en conjunto con nuevas formas de ser. De acuerdo a lo expresado conviene mencionar que los papeles al interior de la familia han transmutado de manera parcial, por ejemplo, las mujeres han accedido al mercado laboral y los hombres son participes de labores domésticas o ha perdido su rol de proveedores económicos, a su vez los hombres ratifican que son la autoridad de la casa y quienes toman las decisiones. Con respecto a lo académico, el contexto escolar y universitario proporciona una visión sobre la necesidad de sensibilizar a estudiantes y docentes sobre el género, aquí se requiere de espacios en donde cuestionen sus comportamientos en relación y con referencia a los otros, se establezca el respeto por la diversidad y se impliquen en la construcción de masculinidades equitativas y no jerárquicas.

La universidad como institución social encargada de transmitir determinados conocimientos, valores y comportamientos debe incluir como quehacer de su ejercicio espacios donde no solo se construya un discurso de equidad de género sino donde se ejerza de manera vivencial y que de paso a procesos reflexivos, críticos y propositivos que cuestionen el papel de mujeres y hombres en la sociedad, de esta manera la educación superior estaría formando jóvenes estudiantes que transgreden un sistema excluyente, violento y discriminatorio.

\section{Referencias}

Ariza, G., Gaviria, S., Geldres, D. y Vargas, R. (2015). Hombres cuidadores de vida: formación en masculinidades génerosensible para la prevención de las violencias hacia las mujeres en Medellín. Revista Colombiana de Psiquiatría, 44(2), 106-114. DOI: https://dx.doi.org/10.1016/j. rcp.2015.01.005

Barba, Á., y Gómez, R. (2016). Percepciones acerca de la masculinidad en un grupo de hombres y mujeres del Área Metropolitana de Bucaramanga, Santander, Colombia. Reflexión Política, 18(36), 212-223.

Bard, G. (2016). Aferrarse o soltar privilegios de género: sobre masculinidades hegemónicas y disidentes. Península, 11(2), 101-122.

Barragán, F. y Gonzales, J. (2007). La construcción de la masculinidad en los contextos escolares. Investigación educativa, 25(1), 167-183.

Boscán, A. (2006). Propuestas críticas para una concepción no tradicional de la masculinidad. Opción, 22(51), 26-49.

Boscán, A. (2008). Las nuevas masculinidades positivas. Utopía y praxis latinoamericana, 13(41), 93-106.

Carrillo, C. D. y Revilla, J. A. (2006). Masculinidad entre padres (madre y padre) e hijos. Masculinidad entre padres (madre y padre) e hijos. Revista de estudios de género: $L a$ ventana, 3 (23), 95-126.

Castaño, J. (2011). Masculinidades y sexualidades de corteros de caña en el municipio de Candelaria, Valle. Sociedady Economía, (21), 221-241.

Cerva, D. (2018). Masculinidad y educación superior: la politización del género. El Cotidiano, Revista de la Realidad Mexicana, (212), 35-45.

Connel, R. (2001) Educando a los muchachos: nuevas investigaciones sobre masculinidad y estrategias de género para las escuelas. Nómadas (Col), (14), 156-171.

Cubillas, M., Abril, E., Domínguez, S., Román, R., Hernández, A. y Zapata, J. (2016). Creencias sobre estereotipos de género de jóvenes universitarios del norte de México. Revista diversitas - perspectivas en psicología, 12(2), 217-230. 
Díaz, E. A. (2014). Filosofía por la paz y la ética del cuidado: reconstrucción y génesis de la agencia para las nuevas masculinidades. Revista Cultura de Guatemala, 35(2), 99-118.

Díez, E. (2015). Códigos de masculinidad hegemónica en educación. Revista Iberoamericana de Educación, 68, 79-98.

Fernández, M. (2012). Indicadores aplicados a la visión dominante de la masculinidad por adolescentes de educación secundaria: la importancia del «deber ser» hombre. Última década (36), 141-162.

Figueroa, J. (2016). Algunas reflexiones para dialogar sobre el patriarcado desde el estudio y el trabajo con varones y masculinidades. Sexualidad, Salud y Sociedad, (22), 221248.

Gallego, G. (2018). Estudios de familia en clave de masculinidades. Estado de la discusión en Colombia. Revista Latinoamericana de Estudios de Familia, 10(2), pp. 30-50.

Garay, B., Vizcarra M. T. y Ugalde, A. I. (2017). Los recreos, laboratorios para la construcción social de la masculinidad hegemónica. Teoría de la Educación. Revista Interuniversitaria, 29(2), 85-209. DOI: 10.14201 / teoredu292185209.

Gordillo, M. (2007). Masculinidad y feminidad: cómo educar en la diferencia. Revista Española de Pedagogía, (238), 447-458.

Guevara, E. (2006). Construcción de la masculinidad en la escuela y la familia en jóvenes universitarios. Psicología para América Latina, (8).

Gutmann, M. (2002). Las mujeres y la negociación de la masculinidad. Nueva Antropología: Revista de Ciencias Sociales, 18(61), 99-118.

Hernández, M. S. (2010). Perfiles de la masculinidad. Fuentes humanísticas, 39, 149-153.

Herrera, P. (2000). Rol de género y funcionamiento familiar. Revista cubana de Medicina General Integral, 16(6), 568-573.

Hervías, V. (2014). Restablecimiento de familias sociables desde el Trabajo Social y las nuevas masculinidades. Revista Prisma Social, (13), 652-704.

Lomas, C. (2013). Orden escolar y (des)orden masculino. Integra Educativa, 6(2), 29-48.

López, I. (2013). La construcción de la masculinidad y su relación con la violencia de género. Comunitania: Revista internacional de trabajo social y ciencias sociales. (5), 61-84.

Macías, R. (2014). Las prácticas corporales para la construcción del actor, la identidad genérica y la(s) masculinidad(es). Cotidiano-Revista de la Realidad Mexicana, 28(184), 7784.

Mardones, K. y Viscarra, M. (2017). Creencias de universitarios del sur de Chile sobre mandatos de género masculinos. Revista de psicología, 26(2), 1-15.
Martínez, C. y Solís, D. (2009). El entorno escolar y familiar en la construcción de significaciones de género y sexualidad en jóvenes de Guadalajara. La ventana, (29),146-183.

Martino, M. (2013). Connel y el concepto de masculinidades hegemónicas: notas críticas desde la obra de Pierre Bourdieu. Estudios Feministas, 21(1), 283-300.

Matamala, M. y Rodríguez, M. (2010). Estudio exploratorio sobre la identidad de género de hombres adolescentes pertenecientes al sector barrio norte de concepción. Última década, (33), 61-84.

Minello, N. (2002). Masculinidad/es: Un concepto en construcción. Nueva Antropología: Revista de Ciencias Sociales, 18(61), 11-30.

Montesinos, R. (2002). La masculinidad ante una nueva Era. El Cotidiano, 18(113), 37-46.

Martín, E., y Echavarría, N. (2017). Narrativas sobre la organización familiar durante el desempleo masculino. Interdisciplinaria: Revista de psicología y ciencias afines = journal of psychology and related sciences. (2), 275294.

Otegui, R. (1999). La construcción social de las masculinidades. Revista Política y sociedad. (32), 151-160.

Ospina, M. (2007). Representaciones sociales de masculinidad y su expresión en el ámbito familiar. Revista académica e institucional de la UCPR, (77).

Otálora, C., y Mora, L. (2014). La construcción de la masculinidad en familias de diferentes contextos sociales. Revista venezolana de estudios de la mujer, 19(43), 103121.

Oliveras, J., Vigo, B., Johnson, J., Rivera Peña, D., y Silva, E. (2018). Reflexiones en torno a la investigación sobre la violencia de género desde las narrativas de investigadoras en formación. Revista Perspectivas, 3(2), pp. 72-85. DOI: https://doi.org/10.22463/25909215.1591

Ramírez, M. y Contreras, S. (2012). Reflexiones en torno a la masculinidad hegemónica en niños de una escuela rural en chile. Psicoperspectivas, 11(1), 158-179. DOI:10.5027/ psicoperspectivas-vol11-issue1-fulltext-163.

Ríos, O. (2015). Nuevas masculinidades y educación liberadora. Intangible Capital, 11(3), 485-507.

Sancho, J. M., Hernández, F., Herraiz, F. y Vidiella, J. (2009). Una investigación narrativa en torno al aprendizaje de las masculinidades en la escuela. Revista Mexicana de Investigación Educativa, 14(43), 1155-1189.

Sandoval, K. (2014). Del dicho al hecho...Las ideologías de género que sustentan las masculinidades hegemónicas. La manzana de la discordia, 9(2), 57-73.

Sanfélix, J. (2011). Las nuevas masculinidades. los hombres frente al cambio en las mujeres. Prisma Social, (7), 220247.

Sagaró, N. M. y Moraga, A. (2015). Creencias acerca del sexo, el género y la masculinidad en adolescentes de distintos niveles de enseñanza. MEDISAN, 19(10), 3012-3019. 
Solano, F. y Rodríguez, S. (2018). Significados sobre la masculinidad construidos por hombres adultos de Buenaventura. La manzana de la discordia, 13(2), 73-90.

Soto, G. (2015). Semblanza histórica de asociaciones de hombres de habla hispana que abogan por la igualdad, resignifica lo masculino y luchan contra la violencia de género. Revista Prisma Social, (13), 944-959.

Téllez, A. y Verdú, A. (2011). El significado de la masculinidad para el análisis social. Revista Nuevas Tendencias en Antropología, (2), 80-10.

Tomasini, M. (2010). Escuela y construcción de identidades de género: una aproximación a la masculinización de los varones en edad pre-escolar. Revista de Psicología, 19 (1), 9-34.

Vázquez, V. y Castro, R. (2009). Masculinidad hegemónica, violencia y consumo de alcoholen el medio universitario. Revista Mexicana de Investigación Educativa, 14(42), 701-719.

Vendrell, J. (2002). La masculinidad en cuestión, Reflexiones desde la antropología. Nueva Antropología: Revista de Ciencias Sociales, 18(61), 31-52.

Vidiella, J., Herraiz, F., Hernández, F. y Sancho, J. (2010). Masculinidad hegemónica, deporte y actividad física. Movimiento, 16(4), 93-115. 